\title{
Sickness certification for mental health problems: an analysis of a general practice consultation database
}

\author{
Christian D. Mallen, Gwenllian Wynne-Jones and Kate M. Dunn \\ Arthritis Research UK Primary Care Centre, Primary Care Sciences, Keele University, Staffordshire, UK
}

\begin{abstract}
Background: Although mental illness remains the leading cause of both sickness absence and incapacity benefit in most high-income countries, little is known about how frequently patients with mental ill-health receive sickness certificates and what conditions are most commonly certified for. This study aims to use general practice consultation data to determine the rate of sickness certification for common mental health problems. Methods: Analysis of a general practice consultation database rates of certification are presented for people consulting with a mental health problem, along with the proportion of these consultations in which a certificate was issued. Results: The highest rates of certification among those consulting with mental health problems occurred for depression, stress-related problems and bereavement. Almost two-thirds of the consultations for bereavement resulted in a sickness certificate being issued. At least one in three mental health consultations resulted in a sickness certificate being issued. Conclusions: Consultation for mental health problems in primary care frequently results in the issuing of a sickness certificate. Further study is urgently needed to provide adequate support systems to enable patients to return to work whenever possible.
\end{abstract}

Key word: mental health; primary care; sickness certification

Received 7 June 2010; accepted 10 November 2010; first published online

3 February 2011

\section{Introduction}

Mental illness remains the leading cause of both sickness absence and incapacity benefit in most high-income countries (Harvey et al., 2009). In the United Kingdom, the role of sickness certification falls predominately on the general practitioner (GP), and until the recent introduction of a modified MED3 ('fit note'), it was difficult for GPs to provide specific recommendations about work when certifying sickness. The Chartered Institute of Personnel and Development reports the rate of UK

Correspondence to: Christian D. Mallen, Arthritis Research UK Primary Care Centre, Primary Care Sciences, Keele University, Staffordshire ST5 5BG, UK. Email: c.d.mallen@ cphc.keele.ac.uk

(C) Cambridge University Press 2011 sickness absence in terms of a percentage of lost working hours (Chartered Institute of Personnel and Development, 2005), estimating sickness absence as $3.5 \%$ of all working hours. This method has several limitations and does not truly reflect the numbers of people absent from work, nor the number of general practice consultations resulting in sickness certification. Furthermore, this method does not accurately ascertain the medical reason for absence and is reliant on occupational databases that are frequently inconsistent. Recent research, using general practice databases, has shown the population rate of sickness certification to be around 102 certificates per 1000 persons per year, with mental health conditions being the most common reason for receiving a sickness certificate (Wynne-Jones et al., 2009). 


\section{Aim}

Mental health conditions are a broad term that encompasses a range of conditions. This study investigates the specific reasons for sickness certification among those with a mental health diagnosis in primary care using consultation data for 2005 .

\section{Methods}

The Keele GP Research Partnership (KGPRP) holds archived data on sickness certification, which can be linked to individual general practice consultations. Fourteen KGPRP practices from North Staffordshire, United Kingdom, contribute data to the archive which included 148176 registered patients aged 20-64 years in 2005. These data sets have been demonstrated to be valid in their recording of sickness certification and consultation data (Porcheret et al., 2004; Jordan et al., 2007; Wynne-Jones et al., 2008). These practices cover a range of areas in terms of deprivation, although generally North Staffordshire is more deprived than England as a whole.

The method for obtaining the sickness certification data has been reported elsewhere (WynneJones et al., 2009). In brief, all sickness certification consultations were downloaded and linked to the corresponding consultation record. The consultation record provides a linked Read code. Read codes are a hierarchy of morbidity, symptoms and process codes that are used to label consultations in UK general practice. For this analysis, consultations with recorded Read codes associated with the Mental Health Chapter were identified. Only patients registered with their practice for the whole of 2005 are included in this analysis.

GPs can issue a number of different types of sickness certificate, depending on when they last saw the patient and the amount of information that should be made available to the patient or the employer. For this study, all types of sickness certificate were grouped together.

Using SPSS, the number of consultations for mental health conditions and the number of certificates issued for each recorded condition were calculated. Rates of certification are presented for people consulting with a mental health problem, along with the proportion of these consultations in which a certificate was issued. The rate of certification was defined as the number of certificates issued for that condition divided by the total number of patient consultations for mental health problems in 2005, separately for men and women. Only conditions in which 20 or more sickness certificates were issued were included in this analysis.

\section{Results}

A total of 11418 consultations occurred for mental health conditions during the study period, with 4116 associated sickness certifications issued. Read codes were matched with 3902 sickness certificates $(95 \%)$. Sickness certificates that were not associated with mental health (eg, non-psychogenic impotence) were excluded, as were sickness certificates that had no associated Read code. Mental health conditions for which less than 20 sickness certificates were issued during the study period are excluded from this analysis.

The most frequent reason for receiving a sickness certificate was depression, followed by stressrelated problems and anxiety. The rate of sickness certification for depression was 123 certificates per 1000 mental health consultations for women and 112 certificates per 1000 mental health consultations for men. Around 3 out of 10 consultations for depression resulted in a sickness certificate being issued, whereas a certificate was issued in more than half of all consultations for bereavement and stress-related problems (Table 1).

In general, although the total number of consultations was higher among women than men, those consultations were equally likely to lead to a sickness certificate being issued. Exceptions were that a higher proportion of consultations resulted in a sickness certificate being issued for women with drug and alcohol problems, and for men with fatigue (although the numbers are small).

\section{Discussion}

Sickness certification for mental health problems managed in primary care is common, with at least one out of every three consultations resulting in a sickness certificate. This estimate may still underrepresent the true rate of certification, as patients 


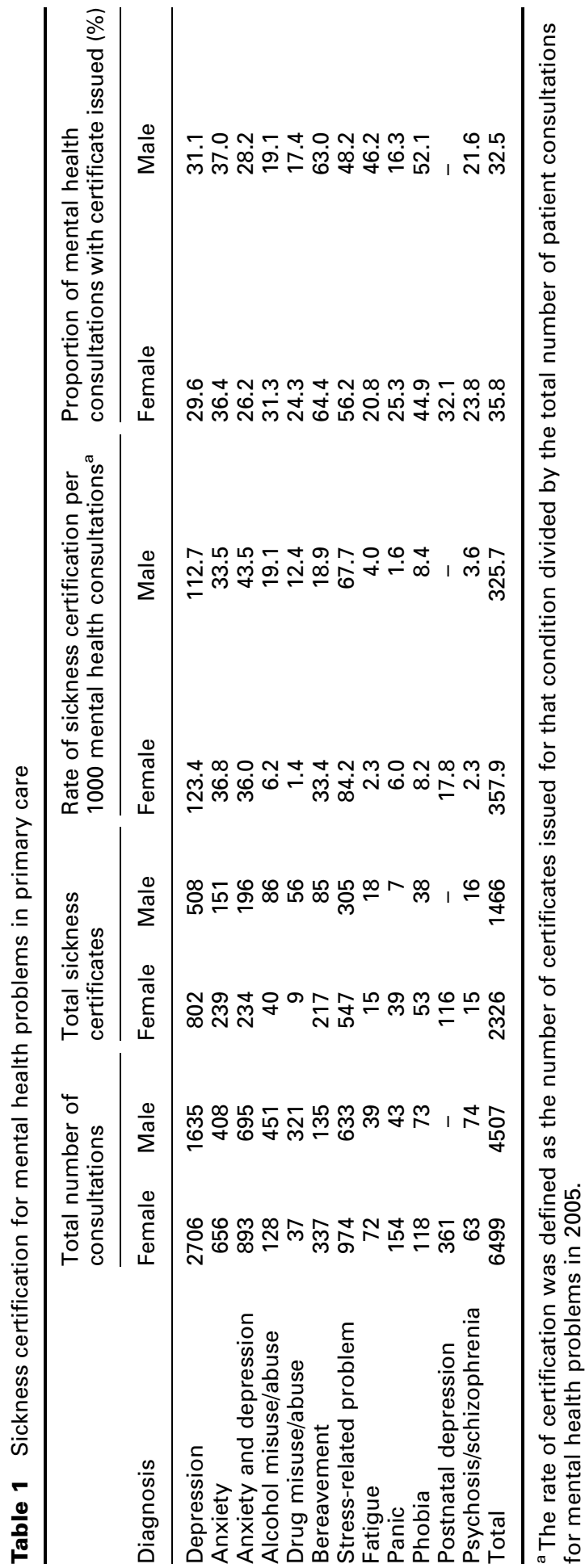

re-consulting with mental health problems may have previously received a sickness certificate. Depression, with or without anxiety symptoms, is the most frequent reason for issuing a sickness certificate, although other conditions such as bereavement and stress-related problems are also commonly certified. Gender differences in sickness certification are apparent, while men have a higher rate of sickness certification for drug/alcohol problems; women are more likely to receive a sickness certificate. The converse is true for fatigue.

This study has some limitations. Although we know the proportion of consultations resulting in a sickness certificate, we do not know the duration of the certificate. Some diagnoses, such as bereavement may reflect acute situations resulting in sickness certification of a short duration, whereas other diagnoses, such as schizophrenia, may be certified for longer periods. Similarly, patients with chronic and severe mental health conditions may have undergone a 'fitness for work' assessment; those incapable of work no longer require sickness certification. This study may also underestimate the true rate of certification, as short duration absence (less than seven days) does not require a medical certificate in the United Kingdom and patients re-consulting for the same problem may still be off work, thus not requiring a repeat certification. This method of assessing sickness certification also does not allow for the collection of additional information about the patient or the costs associated with sickness absence. Combining this approach with other methods, such as occupational records, could help to provide a truer reflection of the current state of sickness certification for mental health problems.

The sickness certification system in the United Kingdom changed in 2010 with the introduction of the 'fit note' - a modified MED3 certificate that gives medical practitioners the opportunity to make specific work-related recommendations to employers. As the impact this will have on levels of certification is unknown, it is hoped that by providing specific recommendations absence can be reduced.

This study uses a primary care diagnosis of the respective mental health disorder, which may not strictly correspond to more formal diagnostic criteria but does reflect everyday clinical general practice. Furthermore, although we used data from 14 large practices, the number of patients 
with less common primary care diagnoses (such as bipolar disorder and personality disorders) is small, making estimation of the rate of certification for these conditions difficult.

\section{Conclusion}

Tackling work absence is currently a national priority, yet, to date accurate information on the rate of, and reasons for sickness certification have been lacking (Black, 2008; Health Work and Wellbeing, 2008). Work is generally deemed to be good for health (Waddell and Burton, 2006) and while issuing a sickness certificate may be appropriate in some circumstances, further study is urgently needed to provide adequate support systems, both within workplace and healthcare settings to enable these patients to return to work whenever possible.

\section{Acknowledgements}

C.D.M. is funded by an Arthritis Research Campaign Career Development Fellowship. K.M.D. is funded through a Research Career Development Fellowship from the Wellcome Trust (083572).

\section{Competing interests}

The authors declare that they have no competing interests.

\section{Authors' contributions}

C.D.M. participated in the design of the study, coded the data, conducted the analysis and drafted the manuscript. G.W-J. participated in the design of the study, coded the data and helped to draft the manuscript. K.D.M. participated in the design of the study and helped to draft the manuscript. All authors read and approved the final manuscript.

\section{References}

Black, C. 2008: Working for a healthier tomorrow. London: TSO. Chartered Institute of Personnel and Development (CIPD). 2005: Absence management: a survey of policy and practice. London: CIPD.

Harvey, S., Henderson, M., Lelliott, P. and Hotopf, M. 2009: Mental health and employment: much work still to be done. British Journal of Psychiatry 194, 201-03.

Health work and wellbeing. 2008: Working for health. Retrieved 5 June 2010 from www.workingforhealth.gov.uk/ Default.aspx

Jordan, K., Clarke, A., Symmons, D., Fleming, D., Porcheret, M., Kadam, U. et al. 2007: Measuring disease prevalence: a comparison of musculoskeletal disease using four general practice consultation databases. British Journal of General Practice 57, 7-14.

Porcheret, M., Hughes, R., Evans, D., Jordan, K., Whitehurst, T., Ogden, H. et al. 2004: Data quality of general practice electronic health records: the impact of a program of assessments, feedback, and training. Journal of the American Medical Informatics Association 11, 78-86.

Waddell, G. and Burton, A. 2006: Is work good for your health and wellbeing? London: TSO.

Wynne-Jones, G., Mallen, C., Main, C. and Dunn, K. 2008: Sickness certification in general practice: a comparison of electronic records with self-reported work absence. Primary Healthcare Research and Development 9, 113-18.

Wynne-Jones, G., Mallen, C., Mottram, S., Main, C. and Dunn, K. 2009: The identification of U.K. sickness certification rates, standardised for age and sex. British Journal of General Practice 59, 510-16. 